\title{
Endogenous Risk AND Growth ${ }^{1}$
}

\author{
JESSE PERLA \\ New York University \\ Christopher Tonetti \\ New York University
}

Working Draft: May 25, 2011

\begin{abstract}
While much of recent growth literature has focused on innovation in the technology frontier, less attention has been paid to the role of the least productive agents in generating growth. We develop an analytically tractable model where growth is created as a positive externality from risk taking by individuals at the bottom of the productivity distribution learning from more productive agents. Heterogeneous firms choose to produce or pay a cost and search for a better opportunity within the economy. Sustained growth comes from the feedback between the endogenously determined distribution of productivity, as evolved by past search decisions, and an optimal forward looking search policy. The growth rate depends on characteristics of the productivity distribution, with a thicker tailed distribution leading to more growth.
\end{abstract}

JEL: D92, E23, 014, O31, O33, O40

Keywords: Endogenous Growth, Productivity Distribution, Firm Heterogeneity, Technology Diffusion, Search

\footnotetext{
${ }^{1}$ We would particularly like to thank Boyan Jovanovic, whose guidance throughout this project has been invaluable. We would also like to thank Jess Benhabib, Ricardo Lagos, Bob Lucas, Ben Moll, Tom Sargent, Gianluca Violante, and Mike Waugh for useful comments and suggestions.

Corresponding Author: jesse.perla@nyu.edu
} 


\section{Introduction}

Productivity growth is a key mechanism for modeling limitless production and technology growth in a resource constrained economy. Many models capture the technology level of the economy as the frontier productivity. However, in any empirical distribution of productivity, there is a large mass of low productivity agents and very few at the frontier of the distribution. ${ }^{1}$ Hence, there are potentially enormous gains in aggregate output from even marginally increasing the productivity of the less productive agents. There are many successful models that investigate the role of a researcher inventing a new frontier technology and mechanisms for the near-frontier firms to adopt it; instead, we will will develop a new model to analyze endogenous changes to the large mass at the lower tail of the productivity distribution.

This paper contributes an analytically tractable endogenous growth mechanism - with an emphasis on the evolution of the productivity distribution-driven by the decisions of the least productive. In the model, heterogeneous firms choose to produce or pay a cost and search for a better opportunity within the economy. While they may get lucky and draw a high productivity, most will make only modest improvements. The expected benefit from risk taking, i.e., the option value of search, will depend on characteristics of the productivity distribution. Search is risky since, ex-post, a firm may regret having searched, depending on the draw, as the value of an improved productivity may be less than the cost of search.

With heterogeneous productivities in a growth model, a key question is which distribution to use for new productivity draws. One standard approach is to use an exogenous distribution or stochastic process for idiosyncratic productivity. While an exogenous distribution gives a clean way to understand the characteristics of the evolving frontier, it may distract from an analysis of the endogenous evolution of the productivity distribution. Instead, a natural candidate for the distribution of new productivity draws is to sample from the existing distribution of productivities itself. When an agent searches, it will copy the ideas of an existing firm in the economy. The result of a meeting is that both agents receive the maximum productivity of the pair. ${ }^{2}$ So not only do the unproductive drive growth, but everyone in the economy does, through the diffusion of technology from the more to the less productive. This leads to the key feature of the model: the distribution of productivity evolves endogenously from past, optimal, forward looking decisions of agents. Aggregate growth is endogenously generated as an externality from rational endogenous risk taking by individuals at the bottom of the productivity distribution. ${ }^{3}$ Not only is growth endogenous, but risk is endogenous, as risk taking through search is an optimal choice.

Section 2 characterizes the dynamic equilibrium of the model, then defines a balanced

\footnotetext{
${ }^{1}$ In Gabaix (2009), firm size is shown to empirically fit a Pareto distribution except for a large mass of small firms. A theoretical relationship between the firm size and productivity distributions is also established. See Aw, Chen, and Roberts (2001) for empirical estimation of productivity distributions, across various industries and over time.

${ }^{2}$ This meet and copy process is similar to Jovanovic and Rob (1989), but does not include the potential for additional spillovers beyond the maximum productivity in the pairwise meeting technology.

${ }^{3}$ Our endogenous evolution of the distribution is most similar to Lucas and Moll (2011) where heterogeneous agents invest in studying to adopt new ideas, and growth is generated as idea adoption evolves the productivity distribution. In contrast to our paper, Lucas and Moll (2011) emphasizes the intensive margin of time dedicated to learning and develops continuous time computational solution techniques.
} 
growth path (BGP) equilibrium, and finally solves for the BGP in closed-form. ${ }^{4}$ We establish a new concept for the BGP of an evolving distribution based on ensuring that the Lorenz curves remain constant over time. Because this evolving distribution is endogenously generated, we can analyze the relationship between characteristics of the productivity distribution and growth. We interpret a thicker-tailed productivity distribution with a higher Gini-coefficient as having more dispersed opportunities. To see the reason why the dispersion of opportunities in the productivity distribution changes the growth rate of the economy, consider an agent sampling from a left-truncated distribution, with the option to continue searching. With a fatter right-tail of the distribution, agents have higher returns to searchsimilar to the effect of a mean-preserving spread of the wage offer distribution in a standard McCall search model. Tempering this mechanism, agents have an incentive to wait for others to push the productivity distribution forward before searching, since search is costly and the value of search is increasing over time. Growth is moderated because infinitesimal agents do not internalize how their search decisions affect the evolution of the distribution.

Solving a planner's problem enables us to investigate this externality, look at the relative social efficiency compared to the competitive equilibrium, and determine if the forces of the model work in similar ways. Section 3 looks at this problem, where the planner chooses which agents search. Unlike in the competitive equilibrium, the planner is able to internalize the effect the search decision has on the evolution of the productivity distribution. Hence, the planner's economy always has higher growth than in the competitive equilibrium. Furthermore, the stronger the degree of inequality in the productivity distribution, the larger the wedge between the planner and the competitive equilibrium's growth rates.

A natural extension is to investigate whether a constrained planner can subsidize search and indirectly adjust the growth rate. In Section 4, we solve the problem of a constrained planner that subsidizes search and satisfies the balanced budget constraint with linear taxes on productive firms. In the spirit of Ramsey problems, the agents operate in a competitive equilibrium where the planner chooses optimal taxes and subsidies subject to a budget constraint. Unlike many tax distortions which affect the elastic labor supply decision of an agent, in this economy proportional taxes impede growth by decreasing the future value of a high productivity draw. However, we find that the planner can overcome this distortion, given parameter restrictions, and still achieve the first best outcome with either a proportional tax or a head tax, while remaining budget feasible.

The broader goal of this paper is to create a minimal - but extensible - model to investigate growth and the evolution of the productivity distribution. While here we focus on fully investigating the growth mechanism in isolation, this model has proven itself amenable to extensions while remaining analytically tractable.

\subsection{Relation to the Literature}

Much of the endogenous growth literature, such as Romer (1990), Grossman and Helpman (1991), Aghion and Howitt (1992), and Klette and Kortum (2004), capture the technology level of the economy as either the total number of differentiated products or as a frontier productivity/quality for each good in the economy. They then investigate how research expands the technology frontier, how this new technology is adopted across the economy,

\footnotetext{
${ }^{4}$ Existence is proven by construction. Uniqueness of the BGP solution is discussed in Appendix B. The only functional form assumptions in the model are constant returns to scale production and homothetic preferences.
} 
and the related intertemporal returns to $R \& D$. Since we do not have a theory of how the frontier expands, our model complements this strain of the growth literature by providing a theory of evolution and diffusion of the entire productivity distribution.

For models interested in the expansion of the technology frontier, it is necessary to choose which distribution to use for new draws in order to understand the returns to R\&D. In Romer (1990) and many other endogenous growth models, the returns to research are proportional to the current stock of research and hence generate geometric growth with a constant research investment. Bental and Peled (1996) instead have searchers drawing new technologies from a Pareto distribution in levels rather than relative improvements. There, the frontier grows if an agent gets a lucky draw above the current frontier, and the economy grows as other firms can copy this technology next period. Kortum (1997) incorporates a mechanism where firms draw from an exogenous distribution of productivities, adjusted for spillovers from the aggregate stock of research. ${ }^{5}$ Our paper provides an alternative approach to the techniques prevalent in the literature: sampling from an endogenously generated distribution allows an analysis of the evolution of the distribution and provides a set of tractable solution techniques.

Another approach in the literature, as in the balanced growth models of Luttmer (2007), Poschke (2009), and Luttmer (2011), is to emphasize how the growth and size distribution of firms contributes to aggregate growth. In these models with firm exit, incumbent firms grow due to an exogenous stochastic productivity process. The productivities of new entrants can be drawn around moments of the productivity distribution. Luttmer (2007) also discusses a variation where new entrants draw from the existing distribution, which is similar to our method of drawing new productivities for searching incumbents. However, in Luttmer (2007) growth is generated by population growth and the Brownian motion with drift which evolves an incumbent's productivity, whereas in our model no firm exit or exogenous stochastic process on idiosyncratic productivity is involved in the growth mechanism.

This paper also broadly fits within the literature on technology diffusion and growth, such as Parente and Prescott (1994), Barro and Sala-i-Martin (1997), Eaton and Kortum (1999), and Benhabib and Spiegel (2005). Much of that literature is interested in cross-country rates of diffusion and rates of catching up to the technology 'leader' to explain differences in growth rates and development, whereas our model contributes a within-country theory of technology diffusion over the entire distribution. Benhabib and Spiegel (2005) analyzes how variations of the technology spillover function can generate lower growth rates if the country is too close to the technology leader. Similarly, we can show that if the productivity distribution has less variation, growth rates will be lower. Since the emphasis is on moving a large number of unproductive enterprises to higher levels of productivity, this paper also fits into the research agenda of Lucas (1988) by attempting to connect a macroeconomic growth model to questions of development.

Finally, this paper emphasizes how the degree of inequality in the economy determines the rate of growth and the strength of the free-riding incentive. An alternative approach, as in Eeckhout and Jovanovic (2002), is to investigate how imperfect spillovers of technology can change the degree of inequality in an economy. There, agents do not copy a technology directly, but rather their production function includes intra-temporal spillovers from the

\footnotetext{
${ }^{5}$ As in our model, Kortum (1997) and Jones (2005) investigate how a Pareto distribution of productivity/idea draws can be consistent with both a stationary distribution of firm characteristics and constant aggregate productivity growth. Similarly, Eaton and Kortum (1999) derives a balanced growth path with a Pareto distribution for the quality of new ideas.
} 
current distribution of technologies. ${ }^{6}$ Eeckhout and Jovanovic (2002) finds that the larger the free-riding incentive, the greater the inequality. Our paper finds that along a balanced growth path, higher inequality increases both the growth rate and the free-riding incentive, while the degree of inequality remains constant.

\section{The Model Economy}

Time is discrete and infinite. There are two types of agents in the economy, consumers and firms. The model admits a representative consumer who simply consumes aggregate production. Firms are heterogeneous, characterized by different productivity levels, $\epsilon$. Given their idiosyncratic productivity, firms have a choice to either search or produce. A firm has access to linear production technology, so if a firm chooses to produce, it creates $\epsilon$ amount of output. If a firm chooses to search, it forgoes production, pays a cost, and copies the technology of some producing firm in the existing economy randomly. This new productivity level will be its idiosyncratic state in the period after search. $F(\epsilon)$ is the cumulative productivity distribution in the economy, which will be the aggregate state variable and evolve over time endogenously in response to firms' actions. Intra-period timing is documented in Figure 1.

When a firm searches, it samples from the existing productivity distribution. Because in equilibrium the unproductive will choose to search, the distribution of productivities evolves by shifting mass from lower to higher productivity levels. Productivity increases over time, despite the lack of an exogenous forcing process. The model is parsimoniously parameterized by a time discount factor, $\beta$, the initial productivity distribution, $F_{0}$, the utility function of the consumer, $u(x)$, and the search cost, $c$. See Figure 2 for intuition on the evolution of the productivity distribution's PDF $f_{t}(\epsilon)$ to $f_{t+1}(\epsilon)$, with a minimum of support $m_{t}$ to $m_{t+1}$.

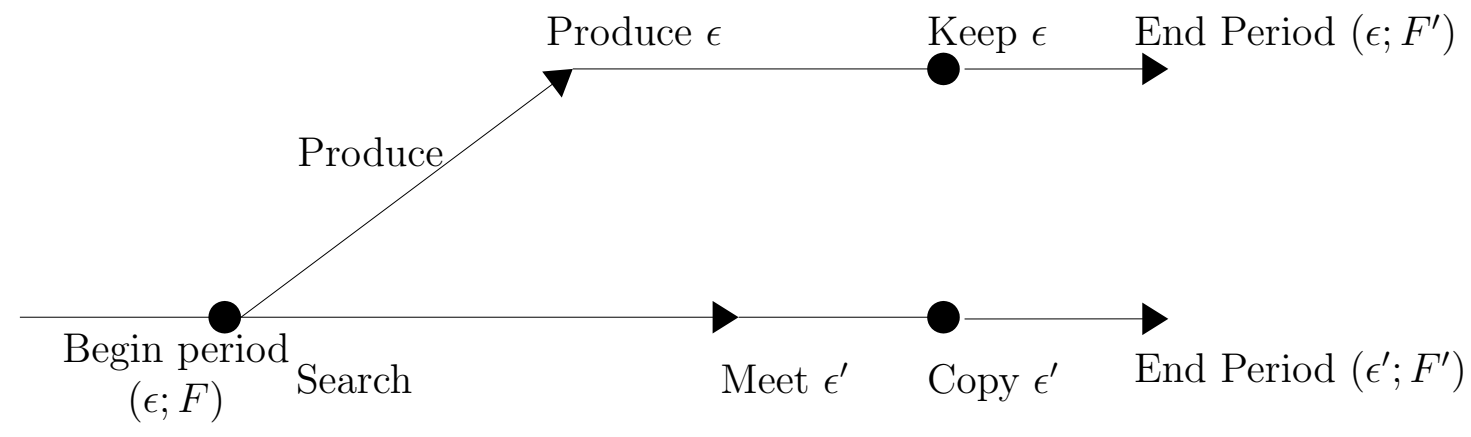

Figure 1: Intra-period Timing

\footnotetext{
${ }^{6}$ Luttmer (2007) investigates how spillovers in the productivity distribution can impact new entrants, while our paper and Eeckhout and Jovanovic (2002) are interested primarily in the effects on incumbents. Also, Luttmer (2007) solves for the tail of the productivity distribution based on model intrinsics along a balanced growth path, but does not focus on analyzing how inequality in the productivity distribution affects growth rates.
} 

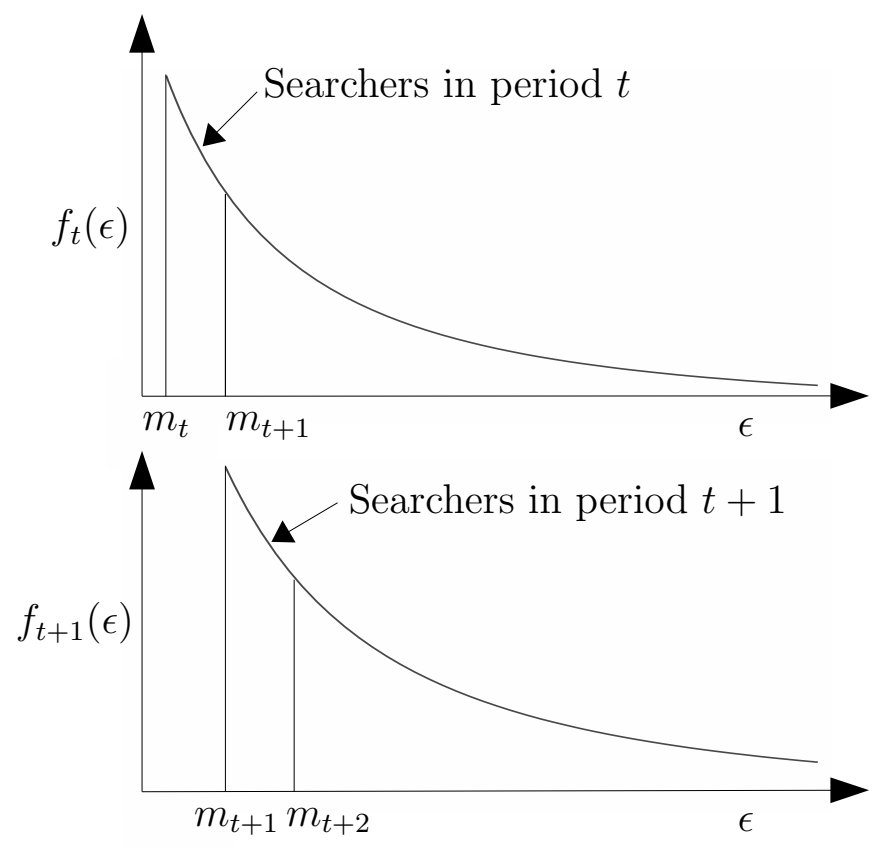

Figure 2: Evolution of the Productivity PDF

\subsection{Consumers}

There exists a continuum of mass one of identical consumers who value the discounted stream of consumption with time separable homothetic preferences represented by period utility, $u(x)$. Consumers are endowed with equal claims to aggregate production at birth. The economy permits a representative consumer who consumes aggregate output, $Y_{t}$, each period. There is no storage technology and no active asset market. Given a utility function $u(\cdot)$ and discount factor $\beta$, the lifetime utility of the representative consumer is given by

$$
\sum_{t=0}^{\infty} \beta^{t} u\left(x_{t}\right), \quad \text { s.t. } x_{t}=Y_{t} \quad \forall t
$$

The implied intertemporal optimization condition yields

$$
\frac{1}{1+r_{t}}=\beta \frac{u^{\prime}\left(x_{t+1}\right)}{u^{\prime}\left(x_{t}\right)}
$$

where $\frac{1}{1+r_{t}}$ is the equilibrium price that ensures there is no active market for claims to aggregate production.

Since there is no aggregate uncertainty, the representative consumer will have a deterministic sequence of consumption. Anticipating a balanced growth path at rate $g$, the sequence of consumption in the economy would be $x_{t+1}=g x_{t}$. Thus, if consumers are risk neutral, with preferences represented by $u(x)=x$, then $\frac{1}{1+r}=\beta$. If consumers have CRRA preferences represented by $u(x)=\frac{x^{1-\sigma}}{1-\sigma}$, with $\sigma>0$, then $\frac{1}{1+r}=\beta g^{-\sigma}$. 


\section{$2.2 \quad$ Firms}

While consumers are kept trivial and simply eat the aggregate output, the important decisions in the economy are made by the firms. There exists a continuum of mass one of risk-neutral, infinitely lived, heterogeneous firms indexed by different productivity levels, $\epsilon$. The starting cumulative productivity distribution in the economy is $F_{0}$ with support $\left\{F_{0}\right\}=$ $\left[m_{0}, \infty\right)$ and PDF $f_{0}$. Firms have access to costless linear production technology such that output equals productivity, i.e., $y=\epsilon$. Since consumers own the firms, a firm's objective is to maximize its discounted stream of output, discounting the future using the interest rate, $r$, determined from the consumer problem.

Given aggregate state $F$ and idiosyncratic state $\epsilon$, firms choose whether to produce or search. The cost of search is forgone production today plus some proportional $\operatorname{cost} c{ }^{7}$ Even though a firm receives a draw higher than their current productivity, the firm may regret the search decision ex-post as the value of an improved productivity may be less than the cost of search. If the firm chooses to search today, it enters next period with a new productivity value drawn from the existing productivity distribution conditional on being a producer, i.e., $\epsilon^{\prime} \sim F(\epsilon \mid \epsilon \geq \operatorname{minimum} \epsilon$ s.t. firm produces), with prime indicating next period. The firm weighs the value of producing versus the value of paying a cost to draw a random productivity level. Formulated recursively, the firm solves:

$$
\begin{aligned}
V(\epsilon, F)=\max _{\{\text {produce,search }\}}\{\epsilon & +\frac{1}{1+r(F, \Gamma)} V\left(\epsilon, F^{\prime}\right), \\
& \left.\quad c \epsilon+\frac{1}{1+r(F, \Gamma)} \int V\left(\epsilon^{\prime}, F^{\prime}\right) d F\left(\epsilon^{\prime} \mid \epsilon^{\prime} \geq h^{e}(F)\right)\right\}
\end{aligned}
$$

s.t. $F^{\prime}=\Gamma(F)$, law of motion for $F$ forecast by agent

where $h^{e}(F)$ is a forecast of the least productive producer's $\epsilon$ this period. The period interest rate, $r$, is a function of consumption growth - itself a function of $F$ and $F^{\prime}$, where $F^{\prime}$ is forecast through $\Gamma$.

One complication in solving this problem is that the productivity distribution, an infinitedimensional object, is a state. Additionally, this distribution is endogenous and depends on the decisions of all firms in the economy, requiring the firms to forecast the evolution of the distribution. The evolution of $F$ is deterministic and perfectly foreseen-made possible by the absence of aggregate risk. We will restrict focus to a rational expectations equilibrium, in which the agents' forecasts match the true evolution of the productivity distribution. ${ }^{8}$

It can be shown - following the arguments of the standard McCall search model - that the solution to this search problem is a reservation value of productivity, $h(F)$, such that all firms with productivity less than $h(F)$ will choose to search and firms with productivity greater than $h(F)$ will choose to produce.

\footnotetext{
${ }^{7}$ The linear search cost $c>-1$ is a multiplier on the opportunity cost of lost production. If $c<0$, then the firm forgoes less than the full period of production when searching. Later, this parameter is manipulated by a planner to subsidize search. Note that if $c>0$, production is negative. Trivial augmentation to this model can ensure positive production without any substantial differences in the results.

${ }^{8}$ Perhaps not surprisingly, when cast as a planner problem where the planner is able to choose which agents search, the problem related to equation 2 has a simple representation (equation 23) and closed form solution (equation 24). More surprisingly, the firm's problem has a simple closed form solution (equation 22) that is similar in structure to the planner's growth rate, but with an economically important difference capturing the externality.
} 


\subsection{Evolution of the Productivity Distribution}

Since the solution takes the form of a reservation productivity function, the firm problem can be greatly simplified, addressing two of the major complications that the endogenously evolving productivity distribution introduces. First, given the existence of a reservation productivity function and the timing assumption that searchers can only match with producers, we can show that the evolution of $F$ is a truncation of the distribution at $h(F) .{ }^{9}$ To see this, trace the evolution of the density for an arbitrary $\epsilon>h(F)$. Given a density $f(\epsilon)$ and a function for this period's search cutoff $h(F)$, the evolution of the density to $f^{\prime}$ evaluated at $\epsilon$ is the density of firms at $\epsilon$ who choose to produce, plus the probability searching firms would draw $\epsilon$ times the mass of searching firms. Formally,

Proposition 1. The evolution of $F$ is a truncation at the reservation value $h(F)$.

Proof.

$$
\begin{aligned}
& f^{\prime}(\epsilon)= \begin{cases}f(\epsilon)+f(\epsilon \mid \epsilon \geq h(F)) F(h(F)) & \forall \epsilon>h(F) \\
0 & \text { otherwise }\end{cases} \\
& =\frac{f(\epsilon)}{1-F(h(F))} \quad \text { support }\left\{f^{\prime}\right\}=[h(F), \infty)
\end{aligned}
$$

Thus, integrating over $[h(F), \epsilon]$, the evolution of the CDF can be characterized by a truncation operator, $\Phi$, and a truncation point function, $h(F)$ :

$$
F^{\prime}(\epsilon)=\Phi(F, h(F))(\epsilon):=\frac{F(\epsilon)-F(h(F))}{1-F(h(F))}
$$

This is a left truncation of the distribution $F$ at $h(F)$.

Furthermore, building on the fact the the evolution of the distribution follows from sequential truncations, only the initial distribution and the last truncation point are necessary to characterize the distribution at any point in time.

Proposition 2. The last truncation point, $m$, is the aggregate state.

Proof.

To see this, truncate the distribution at $m$ to produce the density $f^{\prime}$ and distribution $F^{\prime}$. Then truncate the $F^{\prime}$ distribution again at $m^{\prime}$ to create density $f^{\prime \prime}$. Finally, substitute for $f^{\prime}$ and $F^{\prime}$ to create $f^{\prime \prime}$, with support $\left\{f^{\prime \prime}\right\}=\left[m^{\prime}, \infty\right)$ :

$$
\begin{aligned}
f^{\prime \prime}(\epsilon) & =\frac{f^{\prime}(\epsilon)}{1-F^{\prime}\left(m^{\prime}\right)} \\
& =\left(\frac{f(\epsilon)}{1-F(m)}\right)\left(\frac{1}{1-\frac{F\left(m^{\prime}\right)-F(m)}{1-F(m)}}\right) \\
& =\frac{f(\epsilon)}{1-F\left(m^{\prime}\right)}
\end{aligned}
$$

\footnotetext{
${ }^{9}$ The timing assumption yields the clean truncation of the distribution, with no mass of agents perpetually left behind. While this simplifying assumption was added for tractability and exposition, economically, it represents directed search towards only the productive firms. The primary mechanism for growth in this paper is from the endogenous selection of who searches, rather than the 'selective sampling' of the right hand tail-although directed search does increase the growth rate. See Section 5 for a discussion of extensions that explore this topic.
} 
Yielding a truncation of the original distribution, $F$, at $m^{\prime}$ independent of the original $m$. Since the aggregate state of the model is $F$ and only $m^{\prime}$ is necessary to reconstruct $F$ from $F_{0}$, without loss of generality the last truncation point is the reduced-dimension aggregate state.

Given that the initial condition $F_{0}$ is a fixed parameter, $m$ is the only aggregate state in the firm problem. Additionally, in order to forecast the evolution of the distribution, firms must only forecast a truncation point rule. For notational convenience, define the distribution given that the current state is $\mathrm{m}: F_{m}(\epsilon):=\frac{F_{0}(\epsilon)-F_{0}(m)}{1-F_{0}(m)}$, for $\epsilon \in[m, \infty)$ and $f_{m}(\epsilon):=\frac{f_{0}(\epsilon)}{1-F_{0}(m)}$.

\subsection{Simplified Firm Problem}

We can now write the firm problem with the reduced dimension state and simplified distribution forecast. As before, a firm must decide whether to produce or search. To do this, it compares its productivity to the reservation productivity level, $h(m)$. The firm must also forecast the evolution of $F$, but it knows that $F^{\prime}$ will be a truncation of $F$, making it sufficient to just forecast the truncation point of the economy's productivity distribution, $h^{e}(m) .{ }^{10}$ This truncation point will be the aggregate state next period.

$$
\begin{aligned}
V(\epsilon, m)=\max _{\{\text {produce,search }\}}\{\epsilon & +\frac{1}{1+r\left(m, h^{e}\right)} V\left(\epsilon, h^{e}(m)\right), \\
& \left.\quad-c \epsilon+\frac{1}{1+r\left(m, h^{e}\right)} \int V\left(\epsilon^{\prime}, h^{e}(m)\right) d F_{h^{e}(m)}\left(\epsilon^{\prime}\right)\right\}
\end{aligned}
$$

\subsection{Equilibrium Concepts}

Since we have now shown that the distribution evolves according to a truncation, $\Phi(F, h(F))$, the rational expectations equilibrium requirement for the evolution of the distribution is that the perceived truncation point is the actual truncation point, i.e., $h^{e}(m)=h(m)$. Additionally, we can define aggregate production from the aggregate state and reservation productivity function by integrating over the productivity distribution net the search costs: $Y(m, h):=\int_{h(m)}^{\infty} \epsilon d F_{m}(\epsilon)-\int_{m}^{h(m)} c \epsilon d F_{m}(\epsilon){ }^{11}$

\subsubsection{Recursive Competitive Equilibrium}

With this in mind, we define an equilibrium for a fully dynamic and general economy.

\footnotetext{
${ }^{10} \mathrm{As}$ is standard in the search literature, the solution to this Bellman equation will be a reservation productivity. To prove this, first note that $h^{e}(\cdot)$ is fixed with a finite valued range, hence the agent forecasts a deterministic evolution of the truncation points of the economy, $m_{t}$. Since the value of search is finite for any $h^{e}(\cdot)$, independent of $\epsilon$, and $F_{h^{e}(m)}$ has infinite support, there always exists an $\epsilon$ such that the firm will produce. The proof then follows from the standard solution techniques of search in a non-stationary economy, as in Lippman and McCall (1976).

${ }^{11}$ As in Lagos (2006), aggregate production is calculated as an integral of the output of producing firms, and hence is dependent on properties of the current distribution and searching decisions. Also similar to Lagos (2006), more search would result in higher mean productivity at the macro level, while the period's aggregate production would be lower.
} 
Definition Recursive Competitive Equilibrium

A Recursive Competitive Equilibrium consists of:

1. An initial distribution $F_{0}$ with support $\left\{F_{0}\right\}=\left[m_{0}, \infty\right), m_{0} \geq 0$, and $\operatorname{PDF} f_{0}$

2. A reservation productivity function of the aggregate state $h(m) ; h: \mathbb{R} \rightarrow \mathbb{R}$

3. A truncation point function of the aggregate state $h^{e}(m) ; h^{e}: \mathbb{R} \rightarrow \mathbb{R}$

4. A value function associated with the firm problem $V(\epsilon, m) ; V: \mathbb{R}^{2} \rightarrow \mathbb{R}$

5. An interest rate $r(m) ; r: \mathbb{R} \rightarrow \mathbb{R}$

Such that:

1. Given $r(m), h(m)$ is the reservation productivity function solving the firm problem $\forall \epsilon$, and $V$ is the associated value function

2. Firms' forecasts match the reservation productivity, $h^{e}(m)=h(m)$, so the productivity distribution evolves as expected: $F^{\prime}=\Phi(F, h(F))$

3. Given $h(m), r(m)$ is consistent with the interest rate in equation (1), i.e., $\frac{1}{1+r(m)}=\beta \frac{u^{\prime}(Y(h(m), h))}{u^{\prime}(Y(m, h))}$

A solution $h(\cdot)$ fully characterizes the equilibrium. From $m_{0}=\min \operatorname{support}\left\{F_{0}\right\}$, the recurrence $m_{t+1}=h\left(m_{t}\right)$ generates a sequence of reservation productivities for all $t$. Due to truncation, the sequence of distributions is characterized by the initial distribution and the sequence of reservation productivities, $F_{t}=\Phi\left(F_{0}, m_{t}\right)$, and aggregate production is $Y_{t}=\int_{m_{t+1}}^{\infty} \epsilon d F_{t}(\epsilon)-\int_{m_{t}}^{m_{t+1}} c \epsilon d F_{t}(\epsilon)$. The growth rate, $g_{t}:=\frac{Y_{t+1}}{Y_{t}}$, may diverge or converge depending on the characteristics of $F_{0}$.

As the goal is to fully analyze the growth mechanism and its dependence on model parameters, in this paper we follow the endogenous growth literature and focus on a balanced growth path equilibrium. ${ }^{12}$ First, a condition for the evolution of the productivity distribution under a balanced growth path is established, followed by a construction of a balanced growth path equilibrium with an analytical solution for the growth rate, $g$. We then examine this analytical solution to understand the important forces at work in the model.

\subsubsection{Stationary Distribution}

To define a balanced growth path for scalar variables, we simply require geometric growth with a constant rate $g>1$. Defining a balanced growth path for an evolving distribution requires more care. As the distribution evolves according to sequential truncation, the support of the distribution is certainly changing and the shape of the distribution could potentially change. To introduce the idea of a stationary distribution, loosely speaking, we want a concept that removes the scale of the support and maintains the shape of the distribution. We turn towards Lorenz curves to construct this formally.

\footnotetext{
${ }^{12}$ Conditions for existence of a dynamic equilibrium, convergence of the growth rate, and positive growth are left for future research. In general, numerical methods are required to solve for $h(\cdot)$.
} 


\section{Definition Lorenz Curve of $F$}

$L$, the Lorenz Curve operator for a continuous distribution with CDF $F$ and inverse-CDF (quantile) function, $F^{-1}(z)$, s.t. $z \in[0,1]$ is given by:

$$
L(F)(z)=\frac{\int_{0}^{z} F^{-1}\left(z^{\prime}\right) d z^{\prime}}{\int_{0}^{1} F^{-1}\left(z^{\prime}\right) d z^{\prime}}
$$

The Lorenz Curve characterizes a distribution abstracting from scale. In our definition of a balanced growth path (BGP) equilibrium, we will use the concept of Constant Lorenz Curves to ensure our economy generates a productivity distribution that evolves in a stationary manner in which the normalized shape of the productivity distribution does not change over time. $^{13}$

\section{Definition Constant Lorenz Curves (CLC)}

Let $\Psi$ be an operator with domain and co-domain the set of all CDFs. Let $F_{0}$, the initial argument, be an element of the domain of $\Psi$.

$\Psi$ with $F_{0}$ generates Constant Lorenz Curves if

$$
L\left(F_{0}\right)(z)=L\left(\Psi^{(t)}\left(F_{0}\right)\right)(z), \quad \forall z \in[0,1], t \geq 1
$$

That is, if the sequence of CDFs $\left\{F_{t}\right\}_{t \geq 0}$ generated by starting with $F_{0}$ and repeatedly applying $\Psi$ all have the same Lorenz curve.

A fully dynamic equilibrium definition places no restrictions on the evolution of the productivity distribution, while a stationary equilibrium requires constant distributions. Constant Lorenz Curves ensures that the distribution maintains its shape as it evolves according to some operator $\Psi$ from the initial condition $F_{0}$. In our economy, the evolution operator $\Psi$ will be the truncation operator evaluated at the reservation productivity, $\Phi(F, h(\min$ support $\{F\}))$.

Traditionally, an economy growing along a balanced growth path is a stationary economy when quantities in levels are rescaled to relative values. Since Lorenz curves are a rescaling of a distribution to a CDF evaluated over quantiles, they have a long history in economics and the inequality literature as a way to compare distributions by removing scale. They are therefore a natural candidate for a balanced growth path requirement, where it is important to ensure that all of the moments of the distributions unrelated to scale remain constant. Many weaker candidate balanced growth path requirements with fewer restrictions exist, such as ensuring that the coefficient of variation remains constant. However, these relaxed BGP requirements potentially allow the economy to evolve in a way inconsistent with the spirit of balanced growth, allowing the distribution at different dates to look radically different and potentially limit toward some form of degeneracy. See Appendix B for an alternative scaling requirement on the distribution that is similar to, although slightly stronger than, Constant Lorenz Curves. The Constant Lorenz Curves requirement is preferred because it is economically intuitive and model independent.

\footnotetext{
${ }^{13}$ If the Lorenz curves for two distributions are the same, then the coefficient of variation and the Gini coefficient will also be the same. The converse is not generally true. This is clear, as the Lorenz curve characterizes a distribution (up to scale). See Iritani and Kuga (1983) for details.
} 


\subsubsection{Balanced Growth Path}

We are now prepared to define a balanced growth path competitive equilibrium (BGP CE). Definition BGP Competitive Equilibrium

A BGP Competitive Equilibrium consists of:

1. An initial distribution $F_{0}$ with support $\left\{F_{0}\right\}=\left[m_{0}, \infty\right)$, and PDF $f_{0}$

2. A reservation productivity function of the aggregate state $h(m) ; h: \mathbb{R} \rightarrow \mathbb{R}$

3. A truncation point function of the aggregate state $h^{e}(m) ; h^{e}: \mathbb{R} \rightarrow \mathbb{R}$

4. A value function associated with the firm problem $V(\epsilon, m) ; V: \mathbb{R}^{2} \rightarrow \mathbb{R}$

5. An interest rate $r \in \mathbb{R}$

6. A growth rate $g \in \mathbb{R}$, with $g>1$

Such that:

1. Given $r, h(m)$ is a reservation productivity solving the firm problem $\forall \epsilon$, and $V$ is the associated value function

2. Firms' forecasts match the reservation productivity, $h^{e}(m)=h(m)$, so the productivity distribution evolves as expected: $F^{\prime}=\Phi(F, h($ min support $\{F\}))$

3. Given $g, r$ is consistent with the interest rate in equation (1), i.e., $\frac{1}{1+r}=\beta g^{-\sigma}$

4. The aggregate state, $m$, grows geometrically at a rate $g$. Or equivalently, the reservation productivity is linear in the aggregate state: $h(m)=g m$

5. Production grows at rate $g$, i.e., $Y(h(m), h)=g Y(m, h)$

6. $\Phi(F, h(\min$ support $\{F\}))$ with $F_{0}$ generates Constant Lorenz Curves

The initial distribution must have infinite right tailed support or the economy would not be able to grow indefinitely. ${ }^{14}$ Rational expectations imposes that $h(m)=h^{e}(m)$, so that firms' forecasts of the distribution evolution match the actual evolution. Restricting to cases where $g>1$ ensures our BGP equilibrium has growth. Having production and the aggregate state growing at rate $g$ restricts our BGP equilibrium to be balanced. We require Constant Lorenz Curves to ensure the distribution remains constant in a normalized sense.

As is well known, not every utility function is compatible with balanced growth. Restricting the lifetime utility function to being additive, homothetic, increasing, and quasi-concave implies period utility must be of the constant elasticity of substitution form, representing CRRA preferences. Thus, for the remainder of the paper we use a power utility function, ensuring a constant intertemporal marginal rate of substitution and consistency with balanced growth.

\footnotetext{
${ }^{14}$ A similar assumption for the support of potential improvements is used in papers such as Bental and Peled (1996), Kortum (1997), and Eaton and Kortum (1999). This paper does not model or emphasize the technology frontier, and the majority of searchers end up with only minor improvements. These papers, and other research that emphasizes $\mathrm{R} \& \mathrm{D}$, could provide a better description for the limitless growth of the technology frontier.
} 


\subsection{Solution and Analysis}

Existence of an equilibrium is proved by construction via a guess and check strategy. The first guess is that a Pareto distribution as the initial condition, $F_{0}$, will fulfill the evolution equilibrium requirements. ${ }^{15}$ Let $F_{0}\left(\epsilon ; m_{0}, k\right)=1-\left(\frac{m_{0}}{\epsilon}\right)^{k}$ and $f_{0}\left(\epsilon ; m_{0}, k\right)=k m_{0}^{k} \epsilon^{-k-1}$, with support $\left\{F_{0}\right\}=\left[m_{0}, \infty\right)$. The equilibrium requirement that $h(m)=g m$ is used throughout the following solution.

Define the initial value of search as $V\left(g m_{0}, m_{0}\right)=: m_{0} W$ for some constant $W$. Note that because every firm draws unconditionally from the productivity distribution, the value of search is equal for all searchers: $V\left(\epsilon, m_{0}\right)=m_{0} W$, for $\epsilon \in\left[m_{0}, g m_{0}\right]$. Our second guess is that the value of search grows geometrically from $m_{0} W$ at rate $g$. Using the BGP constraint on the growth of the reservation productivity value, $V(\epsilon, m)=m W$, for $\epsilon \in[m, g m]$. This guess must be verified during the solution process.

The solution strategy is to use the guesses on the distribution and evolution to solve for the constants $(W$ and $g)$ as functions of parameters $\left(m_{0}, k, \beta, \sigma\right.$, and $\left.c\right)$. We then verify that the solution fulfills all of the criteria of a BGP and our guess on the evolution of the value of search. The key to the solution strategy is to use the indifference equations obtained by evaluating the value function at points in the state space that make firms indifferent between searching and producing.

Under certain parameter restrictions, the problem has been solved analytically with the guesses and BGP requirements verified.

Proposition 3. Given the following initial condition and parameter restrictions:

1. The initial productivity distribution, $F_{0}$, is Pareto with $m_{0}>0$ and $k>1$

2. $(1+c) /\left(\frac{k}{k-1}+c\right)<\beta<\min \left\{\left[\frac{1}{1+c}\left(\frac{k}{k-1}+c\right)\right]^{\frac{\sigma-1}{k}}, 1\right\}$

An equilibrium exists according to Section 2.5.3 with the following properties:

1. The growth rate is: $g=\left[\frac{\beta}{1+c}\left(\frac{k}{k-1}+c\right)\right]^{\frac{1}{\sigma-1+k}}>1$

2. The value of search is: $V(\epsilon, m)=\left(\frac{g}{1-\beta g^{1-\sigma}}\right) m, \forall \epsilon \leq g m$

3. The interest rate is: $\frac{1}{1+r}=\beta g^{-\sigma}$

4. The number of agents searching is constant: $S(m)=1-g^{-k} \cdot{ }^{16}$

5. The production of the economy is linear in the aggregate state, and hence grows geometrically: $Y(m)=\frac{k}{k-1}\left[(1+c) g^{1-k}-c\right] m$

Proof.

Appendix A shows that the Pareto guess as the initial distribution fulfills the law of motion, constant proportion searching, geometric growth of aggregate production, and Constant

\footnotetext{
${ }^{15}$ See Appendix B.2 for a proof of the uniqueness of the Pareto distribution for the BGP, given slightly stronger requirements on the balanced growth path evolution of the productivity distribution.

${ }^{16}$ If the proportion of firms searching either grew or shrank monotonically with no limit in $(0,1)$, then there would be no growth in the limit.
} 
Lorenz Curves. The remaining task is to solve for $g$ as a function of parameters such that $h(m)=g m$, where $h(m)=h^{e}(m)$ solves the firm problem.

With $f_{0}\left(\epsilon ; m_{0}, k\right)=k m_{0}^{k} \epsilon^{-k-1}$, the distribution as a function of the aggregate state will be $f_{m}(\epsilon)=k m^{k} \epsilon^{-k-1}$. Using equation 4, plug in $f_{m}$ and the requirement that $h(m)=g m$

$$
V(\epsilon, m)=\max \left\{\epsilon+\frac{1}{1+r} V(\epsilon, g m),-c \epsilon+\frac{1}{1+r} k(g m)^{k} \int_{g m}^{\infty} V\left(\epsilon^{\prime}, g m\right) \epsilon^{\prime-k-1} d \epsilon^{\prime}\right\}
$$

Note that the indifference level of productivity is $\mathrm{gm}$

$$
\begin{aligned}
V(g m, m) & =g m+\frac{1}{1+r} V(g m, g m) \\
& =-c g m+\frac{1}{1+r} k(g m)^{k} \int_{g m}^{\infty} V\left(\epsilon^{\prime}, g m\right) \epsilon^{\prime-k-1} d \epsilon^{\prime}
\end{aligned}
$$

Using the guess that the value of search grows geometrically, $V(g m, m)=m W$, gives two equalities

$$
\begin{aligned}
m W & =g m+\frac{1}{1+r} g m W \\
& =-c g m+\frac{1}{1+r} k(g m)^{k} \int_{g m}^{\infty} V\left(\epsilon^{\prime}, g m\right) \epsilon^{-k-1} d \epsilon^{\prime}
\end{aligned}
$$

Equate the first of the two equalities in equation 10 to get an equation in $W$ and $g$

$$
W=\frac{g}{1-g /(1+r)}
$$

Equate the 2nd equality between equation 10 and equation 11, and splitting the integral

$$
\begin{aligned}
(1+c) g m+\frac{1}{1+r} g m W & =\frac{1}{1+r} k(g m)^{k} \int_{g m}^{g^{2} m} V\left(\epsilon^{\prime}, g m\right) \epsilon^{-k-1} d \epsilon^{\prime} \\
& +\frac{1}{1+r} k(g m)^{k} \int_{g^{2} m}^{\infty} V\left(\epsilon^{\prime}, g m\right) \epsilon^{\prime-k-1} d \epsilon^{\prime}
\end{aligned}
$$

By the decision rule, firms will search next period if $\epsilon \leq g^{2} m$ with value $g m W$

$$
\begin{aligned}
\int_{g m}^{g^{2} m} V\left(\epsilon^{\prime}, g m\right) \epsilon^{\prime-k-1} d \epsilon^{\prime} & =g m W \int_{g m}^{g^{2} m} \epsilon^{\prime-k-1} d \epsilon^{\prime} \\
& =\frac{g m W}{k}(g m)^{-k}\left(1-g^{-k}\right)
\end{aligned}
$$

By the decision rule, firms will produce next period if $\epsilon>g^{2} m$

$$
\begin{aligned}
\int_{g^{2} m}^{\infty} V\left(\epsilon^{\prime}, g m\right) \epsilon^{\prime-k-1} d \epsilon^{\prime} & =\int_{g^{2} m}^{\infty}\left[\epsilon^{\prime}+\frac{1}{1+r} V\left(\epsilon^{\prime}, g^{2} m\right)\right] \epsilon^{\prime-k-1} d \epsilon^{\prime} \\
& =\frac{1}{k-1}\left(g^{2} m\right)^{1-k}+\frac{1}{1+r} \int_{g^{2} m}^{\infty} V\left(\epsilon^{\prime}, g^{2} m\right) \epsilon^{\prime-k-1} d \epsilon^{\prime}
\end{aligned}
$$


Using the indifference equation at the next period, where the aggregate state is $\mathrm{gm}$

$$
\begin{aligned}
V\left(g^{2} m, g m\right) & =g^{2} m+\frac{1}{1+r} g^{2} m W \\
& =-c g^{2} m+\frac{1}{1+r} k\left(g^{2} m\right)^{k} \int_{g^{2} m}^{\infty} V\left(\epsilon^{\prime}, g^{2} m\right) \epsilon^{\prime-k-1} d \epsilon^{\prime}
\end{aligned}
$$

Combining equations 15, 17, and 18 with equation 13 and simplifying yields

$$
(1+c)(1+r) g^{k}=-W+\frac{k}{k-1} g+g\left(1+c+\frac{1}{1+r} W\right)
$$

As $m$ has dropped out of equations 19 and $12, W$ and $g$ are not functions of the aggregate state. Hence, the guess of the functional form $V(g m, m)=W m$ and the equilibrium requirement that $g$ is constant are confirmed.

Combining equation 19 with 12 and solving for $g$ as a function of parameters

$$
g=\left[\frac{1 /(1+r)}{1+c}\left(\frac{k}{k-1}+c\right)\right]^{\frac{1}{k-1}}
$$

Given this fixed $g$, substitute $\frac{1}{1+r}=\beta g^{-\sigma}$ into equation 20 and solve for $g$

$$
g=\left[\frac{\beta}{1+c}\left(\frac{k}{k-1}+c\right)\right]^{\frac{1}{\sigma-1+k}}
$$

The restrictions on parameters come from the requirements that both $g>1$ and $W>0$. For $W>0$, it is necessary and sufficient that $g /(1+r)<1$.

If $c=0$ then

$$
g=\left(\beta \frac{k}{k-1}\right)^{\frac{1}{\sigma-1+k}}
$$

With $c=0$, the interpretation of the constraint $\beta>\frac{k-1}{k}$ is that the discount factor has to be high enough, compared to the search opportunities, to ensure the agents would want to search. If the tale is thinner (larger $k$ ), then agents need to be more patient to ensure search and positive growth. If $\sigma>1$, consumer period utility has an Inada condition and the upper constraint of $\beta<1$ restrains growth. Otherwise, the constraint $\beta<\left(\frac{k}{k-1}\right)^{(\sigma-1) / k}$ ensures that patience or inequality is low enough, compared to the curvature of the utility function, to avoid infinite growth.

Model behavior can be summarized by the derivatives of the growth rate with respect to the parameters. As analytical derivatives are attainable, it is easy to show that $\frac{d g}{d \beta}>0, \frac{d g}{d k}<$ $0, \frac{d g}{d \sigma}<0, \frac{d g}{d c}<0$.

The closed form growth rate provided in Proposition 3 and the above derivatives succinctly capture the connection between all model parameters and the endogenously determined growth rate. This allows a transparent analysis of the relationship of consumers' 
patience, the thickness of the tail of the productivity distribution, and the cost of searching to growth and the evolution of the productivity distribution.

As $\beta$ increases and agents become more patient, growth increases because more value is put on higher future consumption, which yields more search. An increase in search costs, $c$, is a disincentive to search and hence decreases growth. As $\sigma$ increases, $\frac{1}{\sigma}$ decreases, and the firms wish to reflect the lower intertemporal elasticity of substitution of the consumers by reducing search to produce more earlier.

A decrease in $k$ corresponds to an increase in productivity inequality. As $k$ decreases, the Pareto distribution has more weight in the tail and less weight around the minimum of the support. Thus, a searching firm is more likely to obtain a higher productivity in an economy with more inequality. ${ }^{17}$

\section{The Planner Problem}

As has been emphasized previously, in this economy growth is moderated as agents do not internalize how their search decisions affect the evolution of the distribution. We can highlight the magnitude of the externality by solving a social planner problem capable of internalizing the benefit of evolving the productivity distribution through search.

The planner can choose who works and who searches in the economy. It will choose a reservation value, $m^{\prime}$, each period below which individuals will search. Equivalently the planner chooses the growth rate each period $g \geq 1$ s.t. $m^{\prime}=g m$. Set $c=0$ for simplicity, so that aggregate production is just $Y(m)=\int_{g m}^{\infty} \epsilon d F_{m}(\epsilon)$. The planner will attempt to maximize the lifetime utility of the representative agent, who derives utility from consuming aggregate production.

$$
U(m)=\max _{g \geq 1}\left\{\frac{\left(\int_{g m}^{\infty} \epsilon d F_{m}(\epsilon)\right)^{1-\sigma}}{1-\sigma}+\beta U(g m)\right\}
$$

With a particular $F_{0}$, the problem can be solved in closed form by guessing and verifying that $U(m)=-A m^{1-\sigma}$ for some $A>0$. This structure ensures that the optimal choice of $g$ is not a function of $m$ and that the first order condition can be used to solve for $A$ and $g$.

Proposition 4. Given the following initial condition and parameter restrictions:

1. The initial productivity distribution, $F_{0}\left(m_{0}, k\right)$, is Pareto with $m_{0}>0$

2. $\frac{k-1}{k}<\beta<1, \sigma>1$ and $k>\frac{\sigma}{\sigma-1}$. ${ }^{18}$

The planner chooses a constant $g>1$ each period such that

$$
g=\left(\beta \frac{k}{k-1}\right)^{\frac{1}{\sigma-1}}
$$

\footnotetext{
${ }^{17} \mathrm{An}$ informal way to see this this is to note the mean of the Pareto distribution is $\frac{m k}{k-1}$ which is decreasing in $k$, while last period's truncation point, $m$, would also be increasing with more search.

${ }^{18}$ Note that the constraints on the planner's problem are more restrictive than those of the competitive equilibrium. For example, the planner's problem cannot be solved for $\sigma \leq 1$.
} 
Proof.

The proof strategy will be to guess a functional form of the value function and use the first order conditions to determine coefficients. Since we want the growth rate to be state independent, we will also need to show that with our guess, the planner's choice of $g$ is independent of the state $m$. Finally, given the coefficient values, we need to show that the objective function in the maximization problem is globally concave to confirm that our choice for $g$ is the global maximum.

Substituting in the Pareto $F_{0}$ into equation 23 and evaluating the integral

$$
U(m)=\max _{g \geq 1}\left\{\frac{\left(\frac{k}{k-1} g^{1-k} m\right)^{1-\sigma}}{1-\sigma}+\beta U(g m)\right\}
$$

Guess that the form of the solution in the aggregate state is $U(m)=-A m^{1-\sigma}$, where $A>0$.

Substitute the guess into equation 25 and define constant $Q:=\left(\frac{k}{k-1}\right)^{1-\sigma}$

$$
-A m^{1-\sigma}=\max _{g \geq 1}\left\{Q \frac{g^{(k-1)(\sigma-1)}}{1-\sigma} m^{1-\sigma}-\beta A g^{1-\sigma} m^{1-\sigma}\right\}
$$

Using $m>0$, divide by $m^{1-\sigma}$

$$
-A=\max _{g \geq 1}\left\{Q \frac{g^{(k-1)(\sigma-1)}}{1-\sigma}-\beta A g^{1-\sigma}\right\}
$$

Since $m$ has dropped out of the expression, $A$ and $g$ will not be functions of $m$, confirming our guess on the functional form of $U(m)$ and ensuring that $g$ is constant.

Assume, for now, that $g$ is interior and take the first order condition

$$
0=g^{k(\sigma-1)}(k-1) Q-\beta A(\sigma-1)
$$

Solving for $A$

$$
A=\frac{Q(k-1) g^{k(\sigma-1)}}{\beta(\sigma-1)}
$$

Substitute equation 29 into equation 27, dropping the max since this $g$ is the argmax. Solving for g:

$$
g=\left(\beta \frac{k}{k-1}\right)^{\frac{1}{\sigma-1}}
$$

Note that $\beta>\frac{k-1}{k}$ is necessary and sufficient for $g>1$. 
Define the maximization problem's objective, $\Omega(g)$, from equation 27 to get

$$
\Omega(g):=Q \frac{g^{(k-1)(\sigma-1)}}{1-\sigma}-\beta A g^{1-\sigma}
$$

Given the parameter restrictions which ensure that $g$ is interior, to show that the optimal $g$ found in equation 30 is the global maximum, it is sufficient to show global strict concavity of $\Omega(g)$ for any fixed $A>0$ and variable $g>1$. Since $k>1, \sigma>1$, and $A>0$, it can be shown that a necessary and sufficient condition for $\frac{d^{2} \Omega(g)}{d g^{2}}<0$ for all $A>0$ and $g>1$ is $k>\frac{\sigma}{\sigma-1}$.

We can summarize the sensitivity of growth to parameter values with the following derivatives: $\frac{d g}{d \beta}>0, \frac{d g}{d k}<0, \frac{d g}{d \sigma}<0$. The explanation for $\frac{d g}{d \beta}$ and $\frac{d g}{d \sigma}$ is identical to that of the competitive equilibrium. The analysis of $k$ is given in Section 3.1.

Remembering that lower $k$ provide a stronger search incentive, the parameter requirement that $k>\frac{\sigma}{\sigma-1}$ ensures that the value of search is not sufficiently high to dominate the curvature of the utility function.

\subsection{Comparing the Planner to the Competitive Equilibrium}

The solutions in equations 22 and 24 are similar, but the competitive equilibrium solution has an additional $k$ in the exponent. This $k$ in equation 22 decreases the growth rate and should be seen as the penalty for the firms not internalizing the value of search in moving the productivity distribution forward.

The ratio of the planner's first best $g$ to the competitive equilibrium growth rate is:

$$
q=\frac{g_{f b}}{g_{c e}}=\frac{\left(\beta \frac{k}{k-1}\right)^{\frac{1}{\sigma-1}}}{\left(\beta \frac{k}{k-1}\right)^{\frac{1}{\sigma-1+k}}}=\left(\beta \frac{k}{k-1}\right)^{\frac{k}{(\sigma-1)(\sigma-1+k)}}
$$

Note that $q>1$ and that $\frac{d q}{d k}<0$. Hence, the planner always chooses higher growth as it internalizes the effect of search on the productivity distribution. The efficiency wedge between the planner's growth rate and the competitive equilibrium's is higher when productivity inequality is greater. Since the inequality parameter $k$ also summarizes the strength of the externality, the larger the free riding incentive, the larger the wedge.

\section{The Constrained Planner: Subsidies and Taxation}

A natural alternative to the unconstrained planner's problem is to constrain the planner to operate within a competitive equilibrium and to use taxes and subsidies to indirectly influence the search decision. A trade off exists for the constrained planner: Subsidies encourage search through a decrease in the realized search cost, but taxation discourages search by decreasing the future value of higher production. Hence, there exists a potential distortion of growth through the extensive margin. 


\subsection{Firm Problem with Proportional Taxation}

A firm solves its problem given its forecast of a constant taxation and subsidy policy of the planner ( $\tau$ and $s$ respectively).

$$
\begin{aligned}
V(\epsilon, m ; \tau, s)=\max _{\{\text {produce,search }\}}\left\{(1-\tau) \epsilon+\frac{1}{1+r\left(m, h^{e}\right)} V\left(\epsilon, h^{e}(m)\right),\right. \\
\\
\left.s \epsilon+\frac{1}{1+r\left(m, h^{e}\right)} \int V\left(\epsilon^{\prime}, h^{e}(m)\right) d F_{h^{e}(m)}\left(\epsilon^{\prime}\right)\right\}
\end{aligned}
$$

Proposition 5. Given the following initial condition and parameter restrictions:

1. The initial productivity distribution, $F_{0}$, is Pareto with $m_{0}>0$ and $k>1$

2. A perceived constant subsidy from the planner $\tau$ and $s$, where $\tau+s<1$

3. $(1-\tau-s) /\left(\frac{k(1-\tau)}{k-1}-s\right)<\beta<\min \left\{\left[\frac{1}{1-\tau-s}\left(\frac{k(1-\tau)}{k-1}-s\right)\right]^{\frac{\sigma-1}{k}}, 1\right\}$

A solution exists, indexed by $\tau$ and $s$, with the following properties:

1. The truncation point/reservation productivity grows geometrically, $h(m ; \tau, s)=g(\tau, s) m$

2. The growth rate is: $g(\tau, s)=\left[\frac{\beta}{1-\tau-s}\left(\frac{k(1-\tau)}{k-1}-s\right)\right]^{\frac{1}{\sigma-1+k}}>1$.

3. The value of search is: $V(\epsilon, m)=\left(\frac{g(1-\tau)}{1-\beta g^{1-\sigma}}\right) m, \forall \epsilon<g m$

Proof.

The proof follows closely that of the competitive equilibrium without taxation presented in Section 2.6 and is omitted for brevity.

The analysis of this competitive equilibrium is similar to Section 2.6. As expected, $\frac{d g}{d \tau}<0$ and $\frac{d g}{d s}>0$, capturing the meaningful trade off between taxes and subsidies. ${ }^{19}$

\subsection{Constrained Planner Problem with Optimal Linear Taxation}

The planner chooses $s(m)$ and $\tau(m)$ to maximize the utility of the representative consumer subject to a balanced budget constraint. Additionally, the planner is constrained to work within the existing structure of the economy, and thus the growth rate generated must be consistent with the solution to the competitive equilibrium of the firms in Proposition $5 .^{20}$ The solution will require verifying that $\tau$ and $s$ are constant in order to validate the forecasts of the firms.

\footnotetext{
${ }^{19}$ This equilibrium also has similar properties to those in Section 2.6.

${ }^{20}$ This approach is analogous to solving a Ramsey problem, where the first-order conditions of the agents in the Ramsey problem are replaced here with the closed-form growth rate, derived from the indifference equations.
} 
The constrained planner solves ${ }^{21}$

$$
\begin{aligned}
U(m)=\max _{0 \leq \tau<1,0 \leq s}\left\{\frac{\left(\int_{g m}^{\infty} \epsilon d F_{m}(\epsilon)\right)^{1-\sigma}}{1-\sigma}+\beta U(g m)\right\} \\
\text { s.t. } g=\left[\frac{\beta}{1-\tau-s}\left(\frac{k(1-\tau)}{k-1}-s\right)\right]^{\frac{1}{\sigma-1+k}} \\
\tau \int_{g m}^{\infty} \epsilon d F_{m}(\epsilon)=s \int_{m}^{g m} \epsilon d F_{m}(\epsilon) \\
\tau(m), s(m) \text { confirm agent forecasts }
\end{aligned}
$$

Proposition 6. Given the following initial condition and parameter restrictions:

1. The initial productivity distribution, $F_{0}$, is Pareto with $m_{0}>0$

2. $\frac{k-1}{k}<\beta<1, \sigma>1$, and $k>\frac{\sigma}{\sigma-1}$

A solution exists such that:

1. The value function is of the form, $U(m)=-A m^{1-\sigma}$, with $A>0$

2. $\tau, s, g$ are constant

3. An analytical expression for $\tau$ and s exists which achieves the first best solution of the unconstrained planner: $g=\left(\beta \frac{k}{k-1}\right)^{\frac{1}{\sigma-1}}$

Proof.

We use a similar strategy to the proof of Proposition 4. Substituting in the Pareto $F_{0}$ into equation 34 and expanding the integrals:

$$
\begin{aligned}
U(m)=\max _{0 \leq \tau<1,0 \leq s}\left\{\frac{\left(\frac{k}{k-1} g^{1-k} m\right)^{1-\sigma}}{1-\sigma}+\beta U(g m)\right\} \\
\text { s.t. } g=\left[\frac{\beta}{1-\tau-s}\left(\frac{k(1-\tau)}{k-1}-s\right)\right]^{\frac{1}{\sigma-1+k}} \\
\tau=s\left(g^{k-1}-1\right)
\end{aligned}
$$

The objective function in equation 35 is identical to the unconstrained planner's problem in equation 23. The solution method is to assume that the first best is possible, find an

\footnotetext{
${ }^{21}$ The planner can alternatively use a head-tax to finance subsidies. Given a head tax $\tau m$, a firm solves a similar problem to equation 33:

$$
V(\epsilon, m ; \tau, s)=\max \left\{\epsilon-\tau m+\frac{1}{1+r\left(m, h^{e}\right)} V\left(\epsilon, h^{e}(m)\right), s \epsilon-\tau m+\frac{1}{1+r\left(m, h^{e}\right)} \int V\left(\epsilon^{\prime}, h^{e}(m)\right) d F_{h^{e}(m)}\left(\epsilon^{\prime}\right)\right\}
$$

Using the methods of Section 2.6, the solution is $g_{\mathrm{ceh}}(\tau, s)=\left[\frac{\beta}{1-s}\left(\frac{k}{k-1}-s\right)\right]^{\frac{1}{\sigma-1+k}}$.

The planner solves a problem similar to equation 34. The competitive equilibrium constraint becomes $g_{\mathrm{ceh}}(\tau, s)$, and the budget constraint becomes, $\tau m=s \int_{m}^{g m} \epsilon d F_{m}(\epsilon)$. Since $g_{\mathrm{ceh}}$ is independent of $\tau$, the planner has no problem achieving the first best solution while remaining budget feasible.
} 
expression for $\tau$ and $s$ that generates the first best growth rate, and then ensure that it is budget compatible according to equation 37.

Substitute for $\tau$ in equation 36 with equation 37 :

$$
g_{c e}=\left[\frac{\beta}{1-s g^{k-1}}\left(\frac{k}{k-1}\left(1-s g^{k-1}+s\right)-s\right)\right]^{\frac{1}{\sigma-1+k}}
$$

A solution is an $s$ such that $g_{c e}=g_{f b}$, where $g_{f b}$ is from equation 24. Defining a monotonic transformation of this relationship: $\Lambda(s):=\frac{1}{\beta}\left(g_{c e}^{\sigma-1+k}-g_{f b}^{\sigma-1+k}\right)$. Expanding:

$$
\Lambda(s ; g)=\frac{1}{1-s g^{k-1}}\left(\frac{k}{k-1}\left(1-s g^{k-1}+s\right)-s\right)-\frac{g^{\sigma-1+k}}{\beta}
$$

Solving for $s$ such that $\Lambda(s ; g)=0$

$$
s=\frac{1}{g^{k-1}+\frac{1}{(k-1) \frac{g^{\sigma-1+k}}{\beta}-k}}
$$

Given that $g=g_{f b}=\left(\beta \frac{k}{k-1}\right)^{\frac{1}{\sigma-1}}$, this provides a solution for $s$ in terms of model intrinsics. With $s$ from equation $40, g$ from equation 24 and model parameters, equation 37 is used to solve for $\tau$.

$$
\tau=\frac{\left(g^{k}-g\right)\left((k-1) g^{k+\sigma}-\beta g k\right)}{(k-1) g^{2 k+\sigma}+\beta g\left(g-g^{k} k\right)}
$$

Since we used the budget constraint to determine $\tau$, we have a solution if $\tau$ and $s$ fulfill $0 \leq \tau<1,0 \leq s$, and $\tau+s<1$. It can be shown that with the constraint $\beta>\frac{k-1}{k}$, all of these requirements are fulfilled. It can also be shown that the upper limit on $\beta$, from $\beta /(1+r)<1$ in the competitive equilibrium, does not bind at the first best $g$.

\section{Conclusion}

This paper contributes a novel and analytically tractable mechanism for analyzing endogenous growth and the evolution of the productivity distribution. Even though the initial specification of the problem is quite complicated, we show that the state space can be simplified without loss of generality. Even this simplified model is still complicated, because the distribution evolves endogenously, and thus we develop a solution technique that allows us to obtain a closed form expression for the growth rate as a function of intrinsic parameters. The only functional form assumptions used in the model are homothetic preferences and constant returns to scale production. Solving the model, we find that for certain parameter values, the economy grows along a balanced growth path and the distribution of productivities does not become degenerate.

With closed form solutions for both the distribution and growth rate, one can clearly understand all of the forces at work in the model and the strength of the growth mechanism. In particular, higher productivity inequality leads to more growth by fueling risk taking. The greater the dispersion of opportunities, the more risks individuals might be willing to take, even if they fail and need to continue searching. In addition to solving and analyzing 
the model, we contribute Constant Lorenz Curves as a new restriction for the stationary evolution of a distribution along a BGP, discussing and formally deriving its relationship with other potential equilibrium requirements in Appendix B. Using a slightly stronger requirement on the evolution of the distribution, we also prove the uniqueness of the Pareto distribution as the initial condition for the BGP solution.

Solving the social planner's problem, we demonstrate that the competitive equilibrium is inefficient, as agents do not internalize how their search decisions affect the evolution of the distribution. Not only is the first best growth rate always higher than the rate in the competitive equilibrium, but the difference between the two is completely captured by the degree of inequality in the productivity distribution. Furthermore, the stronger the degree of inequality, the larger the effect of the externality and the larger the wedge between the planner and the competitive equilibrium's growth rates. This externality suggests a government policy of using a tax funded program to subsidize shutting down unproductive enterprises. Although a trade off exists for the constrained planner, with subsidies encouraging search and taxation discouraging search, we demonstrate that the first best can be achieved using either head taxes on all agents or proportional taxes on production.

This version of the model is too frugal with parameters to be matched to aggregate growth data or firm micro data. However, the model has shown itself analytically robust to extensions and might later be extended to match empirical income distributions and growth rates.

One key area of future investigation is to solve the model with full dynamics - enabling analysis when the initial value of the productivity distribution is not Pareto. In those cases, the growth rate may not be constant and may converge to infinity or zero. Full dynamics will allow us to investigate how different properties of the productivity distribution affect the growth rate. For example, if the $\mathrm{PDF}$ of $f_{0}$ is non-monotone, growth cycles may exist; or if the thickness of the distribution increases after a given truncation point, the growth rate may substantially increase.

As this stylized version of the model contains neither aggregate nor idiosyncratic shocks to a firm's productivity outside of the search process, it cannot help us understand the relationship between uncertainty, business cycles, welfare, and growth - in closed or open economies. ${ }^{22}$ Adding idiosyncratic shocks to a producing firm's productivity could have two effects. First, the future value of a high productivity draw may be reduced, lessening incentives to search. Second, diffusion could force more agents below the reservation productivity level, causing firms to search that would otherwise wait for the economy to 'catch up' to their productivity levels. Thus, depending on the specification of the idiosyncratic shocks, greater uncertainty could lead to greater growth. Adding aggregate shocks to productivity would allow us to examine the relationship between aggregate production, growth rates, and

\footnotetext{
${ }^{22}$ The model as presented in this paper has perfect consumption insurance and no frictions (such as borrowing constraints) that prevent search for the unproductive. Governments also have no power to change the distribution of opportunities. Hence we cannot conduct experiments with polices that decrease inequality or study the causes and effects of agents unable to benefit from growth. Lucas has argued that the potential welfare improvements from distributional reallocation of consumption goods is inconsequential when compared to the gains from growth. This conjecture deserves rigorous analysis, and variations on our model can relax perfect consumption insurance and introduce frictions. Motivated by Lucas (1987) and the welfare cost of business cycles literature, Cordoba and Verdier (2007) postulates an ad hoc technological frontier equation relating the consumption process to inequality as a constraint in the planner problem. A version of our model with aggregate shocks would provide a micro-foundation to replace this ad hoc equation and endogenously determine the relationship between growth, inequality, and social welfare.
} 
TFP. Greater dispersion of aggregate shocks may increase the long-term growth rate of the economy, even while potentially lowering social welfare.

Work in progress, using the growth mechanism derived here, hopes to provide a better understanding of the interaction between growth, search frictions, and inequality. Instead of having searching firms match a producing firm with certainty, a congestion could occur if too many searching firms try to copy a small set of producing firms. This can be modeled as either searchers drawing from the unconditional distribution of firms or through a matching function. These matching processes introduce a concept of frictional unemployment, where searchers are unable to find a match, and structural unemployment, where firms endogenously shut down to update their technology to the new level of the economy. Finally, moving from a model with a representative consumer to one where the individuals each own a firm and face idiosyncratic unemployment risk due to the matching frictions will provide a theory that jointly determines the consumption and income distributions. 


\section{Appendix A Pareto Family Fulfills Stationarity}

The Pareto with truncations (as defined in Section 2) with PDF and CDF

$$
\begin{aligned}
& f_{m}(\epsilon)=k m^{k} \epsilon^{-k-1} \\
& F_{m}(\epsilon)=1-\left(\frac{m}{\epsilon}\right)^{k}, \quad \text { with support }\left\{F_{m}\right\}=[m, \infty)
\end{aligned}
$$

has the following properties :

Proposition 7. For all $k>1, m_{0}>0, g \geq 1, F_{0}=\operatorname{Pareto}\left(m_{0}, k\right)$ and $F_{m}=$ Pareto $(m, k)$ fulfills:

1. Truncation law of motion

2. Constant Proportion Searching: $S(m)=1-g^{-k}$

3. Production grows at rate $g$ :

$g\left[\int_{g m}^{\infty} \epsilon d F_{m}(\epsilon)-c \int_{-\infty}^{g m} \epsilon d F_{m}(\epsilon)\right]=\int_{g^{2} m}^{\infty} \epsilon d F_{g m}(\epsilon)-c \int_{-\infty}^{g^{2} m} \epsilon d F_{g m}(\epsilon)$

4. Constant Lorenz Curves: $L\left(F_{m}\right)=L\left(F_{g m}\right), \operatorname{Gini}\left(F_{m}\right)=\operatorname{Gini}\left(F_{g m}\right)$

Proof.

The following rely on definitions provided in Section 2.5.3

1. See Section 2

2. $S(m)=\int_{m}^{g m} d F_{m}(\epsilon)=k m^{k} \int_{m}^{g m} \epsilon^{-k-1}=1-g^{k}$, independent of $m$

3. Using the formula for production

$$
Y(m)=\int_{g m}^{\infty} \epsilon d F_{m}(\epsilon)-c \int_{m}^{g m} \epsilon d F_{m}(\epsilon)=\frac{k}{k-1}\left[(1+c) g^{1-k}-c\right] m
$$

4. The inverse CDF, $F_{m}^{-1}(z)=\frac{m}{(1-z)^{1 / k}}$, is linear in $m$. Hence the Lorenz curve is independent of $\mathrm{m}$, and the Gini follows from the the Lorenz Curve of the Pareto. 


\section{Appendix B Alternative BGP Requirements for Distribution Evolution}

\section{B.1 Requirements on Distribution Stationarity}

Maintaining the following assumptions throughout this section:

- $F_{0}$ is a CDF with support $\left\{F_{0}\right\}=\left[m_{0}, \infty\right)$ for $m_{0}>0$, an initial state

- $F_{0}$ has a differentiable PDF $f_{0}$

- $F_{0}$ has a differentiable inverse-CDF (quantile) function, $F_{0}^{-1}(z), \forall z \in[0,1]$

- $\phi: \mathrm{PDF} \times \mathbb{R}$ to the set of PDFs

- $\Xi: \mathrm{CDF} \times \mathbb{R}$ to the set of CDFs

- $G \geq 1 .^{23}$

We use Constant Lorenz Curves as our BGP equilibrium restriction on the evolution of the productivity distribution. In Section 2.5.2, we discussed that there are many potential equilibrium restrictions and we defended why CLC is a better choice than weaker restrictions. Here we argue that CLC is also a better concept than other stronger restrictions, in that it is more general and less model specific.

In the following section we define Positive Scale Invariance, which is a stronger restriction related to multiplicative scaling requirements more commonly seen in the literature. Additionally, we define Strongly Constant Lorenz Curves (SCLC) as an alternative BGP requirement that is very similar to, but slightly more restrictive than, Constant Lorenz Curves. CLC only requires that the distribution remains stationary when evaluated along the discrete truncation points of the balanced growth path. There may exist cases where CLC only holds for an initial distribution with a particular sequence of growth rates and period lengths. SCLC is stronger in that it requires both CLC and that the aggregate state can be simplified into a scalar that grows geometrically. Furthermore, SCLC requires constant Lorenz curves for any scalar aggregate state, even those not realized along the BGP. This requirement on an off-equilibrium evolution path seems excessive. Similarly, multiplicative scaling requirements are very model specific and overly restrictive as a general theory of BGP distribution evolution, as, for example, they would rule out economies with additive growth.

Linking potential equilibrium restrictions using Lorenz curves to those using scaling requirements, we prove that Positive Scale Invariance implies SCLC. Furthermore, we prove that they are equivalent if a particular geometric growth requirement is satisfied. Not only does this Appendix argue that CLC is more general and more economically natural than multiplicative scale requirements, it also provides a relationship between more commonly used direct scaling requirements and our preferred Lorenz curve based equilibrium restriction.

Finally, under SCLC (or Positive Scale Invariance, by equivalence), we prove that the Pareto distribution is the only initial distribution that can satisfy the BGP requirements of the model presented in this paper.

\footnotetext{
${ }^{23}$ The aggregate state of the economy is $m_{0} G$ for an evolving $G$. For example, $G$ could be the product of growth rates up to a period in the model $G=g_{0} \times g_{1} \times \ldots \times g_{t}$ for a sequence of growth rates $\left\{g_{t}\right\} \geq 1$.
} 
Definition Strongly Constant Lorenz Curves (SCLC)

Let $F_{0}$, the initial argument, be an element of the range of $\Xi$. Then $\Xi$ with $F_{0}$ generates Strongly Constant Lorenz Curves from the initial state $m_{0}$ if

$$
L\left(F_{0}\right)(z)=L\left(\Xi\left(F_{0}, m_{0} G\right)\right)(z), \quad \forall z \in[0,1], G \geq 1
$$

While Constant Lorenz Curves is appropriate for an arbitrary evolution of the distribution, SCLC requires that that the evolution of the distribution has no history-dependence and can be summarized with a scalar (or more generally, a finite set of scalars) and an initial condition. This model, with $\Xi\left(F_{0}, m_{0} G\right)(\epsilon):=F_{m_{0} G}(\epsilon)$ from the notation in Section 2.3, has no history dependence as proved in Proposition 2. However, since the stationary evolution of a distribution should be a general concept not connected to this particular model, and should not require a strong condition such as history-independence, Constant Lorenz Curves is used throughout the paper.

An alternative BGP requirement for the evolution of the productivity distribution is to ensure that the PDF can be pointwise scaled. The intuition is that quantiles such as the median or 10th percentile all grow at the same rate, $g$.

Definition Positive Scale Invariance

Given the maintained assumptions and that $f_{0}$ is in the range of $\phi, f_{0}$ with $\phi$ satisfies Positive Scale Invariance from the initial state $m_{0}$ if

$$
\phi\left(f_{0}, m_{0} G\right)(\epsilon)=\frac{1}{G} f_{0}(\epsilon / G), \quad \text { for all } G \geq 1, \epsilon \in \operatorname{support}\left\{\phi\left(f_{0}, m_{0} G\right)\right\}
$$

Denoting $f_{G}(\epsilon):=\phi\left(f_{0}, m_{0} G\right)(\epsilon)$, this expression becomes

$$
f_{G}(\epsilon)=\frac{1}{G} f_{0}(\epsilon / G), \quad \text { for all } G \geq 1, \epsilon \in \operatorname{support}\left\{f_{G}\right\}
$$

Note that the truncation is relative to $m_{0}$. This requirement can also be written with the $\mathrm{CDF}$ for $f_{G}, F_{G}$, or the quantile function, $F_{G}^{-1} \cdot{ }^{24}$

Proposition 8. Given the maintained assumptions, $f_{0}$ with $\phi$ fulfilling Positive Scale Invariance is equivalent to:

- $F_{G}(\epsilon)=F_{0}(\epsilon / G)$

- $F_{G}^{-1}(z)=G F_{0}^{-1}(z)$

Proof.

Proof of the first equivalence comes from a differentiation of the CDF, or a change of variables and integration of $f_{0}$ in the converse. Proof of the second is done by taking inverse functions and using Positive Scale Invariance on the CDF.

Proposition 9 shows that this technical requirement on the scaling of the distributions is equivalent to Strongly Constant Lorenz Curves when the aggregate state grows geometrically.

\footnotetext{
${ }^{24}$ The $f_{G}$ notation for a general operator $\phi$, which embeds scaling relative to an initial condition $m_{0}$, should not be confused with the truncation notation used in Section 2.3.
} 
Proposition 9. Positive Scale Invariance implies Strongly Constant Lorenz Curves. Moreover, if the economy is growing such that $\mathbb{E}\left[F_{G}\right]=G \mathbb{E}\left[F_{0}\right]$, then Strongly Constant Lorenz Curves is equivalent to Positive Scale Invariance. ${ }^{25}$

Proof.

Positive Scale Invariance $\Longrightarrow$ Strongly Constant Lorenz Curves

Take the formula of the Lorenz curve, $L(F)(z)=\frac{\int_{0}^{z} F^{-1}\left(z^{\prime}\right) d z^{\prime}}{\int_{0}^{1} F^{-1}\left(z^{\prime}\right) d z^{\prime}}$, and equate:

$$
\frac{\int_{0}^{z} F_{0}^{-1}\left(z^{\prime}\right) d z^{\prime}}{\int_{0}^{1} F_{0}^{-1}\left(z^{\prime}\right) d z^{\prime}}=\frac{\int_{0}^{z} F_{G}^{-1}\left(z^{\prime}\right) d z^{\prime}}{\int_{0}^{1} F_{G}^{-1}\left(z^{\prime}\right) d z^{\prime}}
$$

Using Positive Scale Invariance, $F_{G}^{-1}(z)=G F_{0}^{-1}(z)$, the $G^{\prime} s$ drop out of the right hand side for any $G \geq 1$.

\section{Strongly Constant Lorenz Curves $\Longrightarrow$ Positive Scale Invariance:}

The formula for Strongly Constant Lorenz Curves implies

$$
\int_{0}^{z} F_{G}^{-1}\left(z^{\prime}\right) d z^{\prime}=\frac{\int_{0}^{1} F_{G}^{-1}\left(z^{\prime}\right) d z^{\prime}}{\int_{0}^{1} F_{0}^{-1}\left(z^{\prime}\right) d z^{\prime}} \int_{0}^{z} F_{0}^{-1}\left(z^{\prime}\right) d z^{\prime}
$$

Differentiate this expression with respect to $z$ to get

$$
F_{G}^{-1}(z)=\frac{\int_{0}^{1} F_{G}^{-1}\left(z^{\prime}\right) d z^{\prime}}{\int_{0}^{1} F_{0}^{-1}\left(z^{\prime}\right) d z^{\prime}} F_{0}^{-1}(z)
$$

Note that these integrals are the expectations of $F_{G}$ and $F_{0}$ respectively. Using the assumption $\mathbb{E}\left[F_{G}\right] / \mathbb{E}\left[F_{0}\right]=G$

$$
F_{G}^{-1}(z)=G F_{0}^{-1}(z)
$$

\footnotetext{
${ }^{25}$ For example, this expectation relationship holds if the aggregate state and aggregate output of the economy grow geometrically.
} 


\section{B.2 Uniqueness of the Pareto Distribution}

The following proposition proves that under the SCLC requirement, the Pareto distribution is the unique initial condition which can fulfill the BGP equilibrium. For clarity of exposition, the following proof of uniqueness assumes, without loss of generality, that Positive Scale Invariance holds for $G>1-\delta$ for some infinitesimal $\delta>0$.

Proposition 10. Given the maintained assumptions, the Pareto distribution is the only distribution which can satisfy Positive Scale Invariance with the truncation operator.

Proof.

Let $\phi$ be the truncation operator

$$
\phi\left(f_{0}, m_{0} G\right)(\epsilon):=\frac{f_{0}(\epsilon)}{1-F_{0}\left(m_{0} G\right)}, \text { for } \epsilon \in\left[m_{0} G, \infty\right),
$$

From Positive Scale Invariance

$$
\phi\left(f_{0}, m_{0} G\right)(\epsilon)=\frac{1}{G} f_{0}(\epsilon / G)
$$

Combining the two relations

$$
\begin{aligned}
f_{0}(\epsilon / G) & =\frac{G f_{0}(\epsilon)}{1-F_{0}\left(m_{0} G\right)} \\
f_{0}(\epsilon / G) & \propto f_{0}(\epsilon), \quad \text { a power law relationship }
\end{aligned}
$$

Differentiate both sides of equation B.8 with respect to $G$

$$
\frac{-d f_{0}(\epsilon / G)}{d \epsilon} \frac{\epsilon}{G^{2}}=\frac{\left(1-F_{0}\left(G m_{0}\right)+G m_{0} F_{0}^{\prime}\left(G m_{0}\right)\right)}{\left(F_{0}\left(G m_{0}\right)-1\right)^{2}} f_{0}(\epsilon)
$$

Evaluate at $G=1$ (w.l.o.g.). Note that $F_{0}^{\prime}(m)=f_{0}(m)$, and $F_{0}\left(m_{0}\right)=0$ from the support of $F_{0}$

$$
\frac{d f_{0}(\epsilon)}{d \epsilon} \epsilon=-\left(1+m_{0} f_{0}\left(m_{0}\right)\right) f_{0}(\epsilon)
$$

This is an ordinary differential equation in $\epsilon$. For any positive valued initial condition, the only solution is the Pareto distribution. If we arbitrarily choose the initial condition to be $f\left(m_{0}\right)=\frac{k}{m_{0}}$, then the particular solution of the differential equation matches the parameterization for a $\operatorname{Pareto}\left(m_{0}, k\right)$ used in this paper.

$$
f_{0}(\epsilon)=k m_{0}^{k} \epsilon^{-(k+1)}
$$




\section{References}

Aghion, P., And P. Howitr (1992): "A Model of Growth Through Creative Destruction," Econometrica, 60(2), 323-51.

Aw, B. Y., X. Chen, And M. J. Roberts (2001): "Firm-level evidence on productivity differentials and turnover in Taiwanese manufacturing," Journal of Development Economics, $66(1), 51-86$.

Barro, R. J., and X. Sala-I-Martin (1997): "Technological Diffusion, Convergence, and Growth," Journal of Economic Growth, 2(1), 1-26.

Benhabib, J., And M. M. Spiegel (2005): "Human Capital and Technology Diffusion," in Handbook of Economic Growth, ed. by P. Aghion, and S. Durlauf, vol. 1 of Handbook of Economic Growth, chap. 13, pp. 935-966. Elsevier.

Bental, B., And D. Peled (1996): "The Accumulation of Wealth and the Cyclical Generation of New Technologies: A Search Theoretic Approach," International Economic Review, $37(3), 687-718$.

Cordoba, J. C., And G. Verdier (2007): "Lucas vs. Lucas: On Inequality and Growth," IMF Working Paper, 07(17).

Eaton, J., And S. Kortum (1999): "International Technology Diffusion: Theory and Measurement," International Economic Review, 40(3), 537-570.

Eeckhout, J., and B. Jovanovic (2002): "Knowledge Spillovers and Inequality," American Economic Review, 92(5), 1290-1307-61.

Gabaix, X. (2009): "Power Laws in Economics and Finance," Annual Review of Economics, $1(1), 255-294$.

Grossman, G. M., and E. Helpman (1991): "Quality Ladders in the Theory of Growth," The Review of Economic Studies, 58(1), 43-61.

Iritani, J., And K. Kuga (1983): "Duality Between the Lorenz Curves and the Income Distribution Functions," The Economic Studies Quarterly, 34(1), 9-21.

Jones, C. I. (2005): "The Shape of Production Functions and the Direction of Technical Change," The Quarterly Journal of Economics, 120(2), 517-549.

Jovanovic, B., And R. Rob (1989): "The Growth and Diffusion of Knowledge," Review of Economic Studies, 56(4), 569-582.

Klette, T. J., And S. Kortum (2004): "Innovating Firms and Aggregate Innovation," Journal of Political Economy, 112(5), 986-1018.

Kortum, S. S. (1997): "Research, Patenting, and Technological Change," Econometrica, 65(6), 1389-1420.

Lagos, R. (2006): "A Model of TFP," Review of Economic Studies, 73(4), 983-1007. 
Lippman, S. A., And J. J. MCCAll (1976): "Job Search in a Dynamic Economy," Journal of Economic Theory, 12(3), 365 - 390.

Lucas, R. E. (1987): Models of Business Cycles. Blackwell.

(1988): "On the Mechanics of Economic Development," Journal of Monetary Economics, 22(1), 3-42.

Lucas, R. E., And B. Moll (2011): "Knowledge Growth and the Allocation of Time," mimeo.

Luttmer, E. G. J. (2007): "Selection, Growth, and the Size Distribution of Firms," The Quarterly Journal of Economics, 122(3), 1103-1144.

coming.

(2011): "Technology Diffusion and Growth," Journal of Economic Theory, forth-

Parente, S. L., And E. C. Prescott (1994): "Barriers to Technology Adoption and Development," The Journal of Political Economy, 102(2), 298-321.

Poschke, M. (2009): "Employment Protection, Firm Selection, and Growth," Journal of Monetary Economics, 56(8), 1074-1085.

Romer, P. M. (1990): "Endogenous Technological Change," Journal of Political Economy, 98(5), S71-102. 